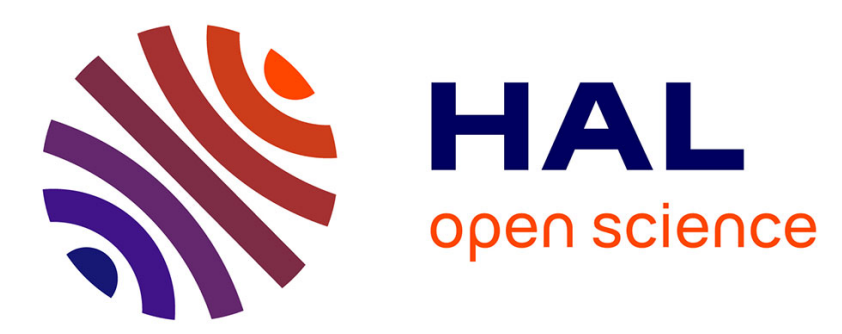

\title{
Settling of mineral aqueous suspensions. Classification and stability prediction by neural networks
}

\author{
Remy Vie, Anne Johannet, Nathalie Azema
}

\section{To cite this version:}

Remy Vie, Anne Johannet, Nathalie Azema. Settling of mineral aqueous suspensions. Classification and stability prediction by neural networks. Colloids and Surfaces a-Physicochemical and Engineering Aspects, 2014, 459, pp.202-210. 10.1016/j.colsurfa.2014.07.005 . hal-02914345

\section{HAL Id: hal-02914345 \\ https://hal.science/hal-02914345}

Submitted on 1 Jun 2021

HAL is a multi-disciplinary open access archive for the deposit and dissemination of scientific research documents, whether they are published or not. The documents may come from teaching and research institutions in France or abroad, or from public or private research centers.
L'archive ouverte pluridisciplinaire HAL, est destinée au dépôt et à la diffusion de documents scientifiques de niveau recherche, publiés ou non, émanant des établissements d'enseignement et de recherche français ou étrangers, des laboratoires publics ou privés. 


\title{
Settling of mineral aqueous suspensions. Classification and stability prediction by neural networks
}

\author{
Rémy Vié, Anne Johannet, Nathalie Azéma* \\ Centre des Matériaux des Mines d'Alès Ecole des Mines d'Alès, 6 avenue de Clavières, 30319 Alès Cédex, France
}

\section{H I G H L I G H T S}

- Classification of mineral aqueous suspensions.

- Use neural networks (ANN) to determine prevailing interparticle interactions.

- Propose a method based on ANN to estimate stability regarding sedimentation.
G R A P H I C A L A B S T R A C T

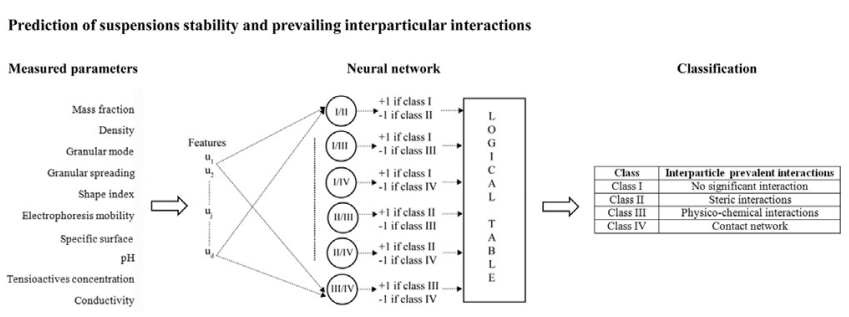

\section{A B S T R A C T}

Aqueous mineral suspensions and pastes are greatly used in industry and waste treatment processes. But unfortunately due to their inherent complexity (numerous parameters to consider and non-linearity of temporal behaviour), their physicochemical stability, controlled by their dispersion state, is difficult to predict. A way to have a better understanding of these systems is to apprehend stability by studying settling behaviour of suspensions in function of solid concentration and interparticle interactions.

To this purpose, previous works on settling optical analysis were used in addition to rheological approach, to determine in some mineral systems, a suspension typology in function of solid mass frac-tion and predominant particle interaction. In order to generalize this work to various mineral aqueous suspensions, a modelling study is proposed in this paper. The aim of this work is to predict the stability of mineral suspensions based on a specific index: phase separation index (PSI) previously established using 10 discriminating parameters currently measured in industrial and academic areas. Because of their well-known ability to model non-linear processes, a neural networks-based procedure was used to classify the settling behaviour in four classes: diluted, concentrated (cohesive and non cohesive) and solid suspension. The method was proved to be very efficient, delivering $100 \%$ of good classification on various sets of test sets up to $60 \%$ of the database allowing thus to predict the suspension stability for applications such as inks, paints, cosmetics. In this research, the predominant influence of mass fraction parameter was showed.

Keywords:

Classification

Stability

Mineral suspension

Neural networks

Settling

\section{Introduction}

Aqueous mineral suspensions and pastes are commonly used in several industrial areas or waste treatment process. Their

\footnotetext{
* Corresponding author. Tel.: +33 0466785356.

E-mail address: nathalie.azema@mines-ales.fr (N. Azéma).
}

macroscopic behaviours such as rheology or physico-chemical stability must be studied to control processes and material properties. However, this control may be difficult because their behaviours depend on various parameters such as solid concentration, particle size, shape or hydrodynamic and interparticle forces.... These latter depend also on a great number of parameters which are linked to the solid or liquid phase characteristics, or particle/liquid interphase. Many rheometry studies showed that, suspensions 
behaviour is generally non linear [1,2] with regards to time. These works distinguished three types of suspensions: diluted, concentrated and solid suspensions depending on prevalent interparticle interactions. The multiplicity of systems leads recent studies to use mathematical tools in order to predict, with few experiments and parameters, the settling behaviour of suspensions [3,4]. In the same spirit, a global method of physico-chemical stability analysis, suitable for classification of kaolin and glass beads suspensions as function of interparticle interactions was proposed in previous works $[5,6]$. They were based on settling measurements carried out using a Turbiscan MA 2000 optical analyser [7]. The authors established a novel index: the phase separation index (PSI), which can be used to a broad range of solid concentrations. The evolution of this index versus the mass solid fraction makes the classification possible as a function of the prevalent type of interparticle interactions. Four classes were derived from the PSI, which can be interpreted in terms of stability behaviour.

To complete and attempt to generalize these studies to all mineral pastes and suspensions, the current work presents an approach based on two steps: the first one study various agglomerated or dispersed suspensions and establishes classification of behaviour based on the PSI index. The second step attempts to generalize the classification and to predict dispersed systems stability as a function of 10 parameters (as particle dimension, density or $\mathrm{pH}$ ) by a neural networks approach. Neural networks were chosen for their ability to apprehend complex functions and their two fundamental properties: universal approximation and parsimony [8]. Furthermore, among the black-box based modelling tools (SVM, others statistical models), they have the capability to be adjusted to the complexity of the underlying function to identify [9], using a unique formalism, which is not for example the case of the SVM. After the implementation of the neural model, because of its simplicity in operational mode, and low cost, the second phase of the method could of a great interest for industrial sectors as inks, paints, cosmetics, ....

The paper is organized as follows: after the presentation of materials and methods, and PSI calculation in Section 2, a brief overview of neural networks definitions and properties will be proposed in Section 4. The approach of classification will then be presented. Finally the efficiency of the classification will be proved. The conclusion will then be given assuming the real interest of the method as well for industrial than academic users of suspensions.

\section{Materials and method}

Suspension settling depends strongly on interparticle forces which can be modified at some critical volume fraction $\left(\phi_{v}\right)$. They affect hydrodynamic interactions via the particle drag and mesoscopic organization as size or shape of agglomerates which determine the settling behaviour and sediment structure. In order to propose a general analysis method applicable to a broad range of systems, a phase separation index (PSI) has been proposed $[5,6]$. This index (Eq. (1) in Section 2.3) is calculated from sedimentation measurements obtained with an optical analyser of concentrated suspensions, the Turbiscan MA2000 from the Formulaction Company.

\subsection{Procedure of samples preparation}

Samples were prepared from dry powder suspended in deionized water until a total weight of $30 \mathrm{~g}$. Suspensions were stirred during $15 \mathrm{~min}$ with a constant speed in order to obtain a homogenous medium. Powders used are kaolin (Prolabo-CAS number 1332-58-7), aluminium hydroxide (Martinswerk) and glass microbeads of various sizes (Potters Europe). Alumina was prepared using aluminium hydroxide treated at $1200^{\circ} \mathrm{C}$ in order to obtain $\alpha$ alumina [10].

\subsection{Settling optical analysis}

The variation in suspension stability as a function of mass fraction was studied at room temperature $\left(21^{\circ} \mathrm{C}\right)$ using the dispersion optical analyser, Turbiscan MA 2000. Although the volume fraction is decisive in theoretical development, the mass fraction has been employed in this work for obvious practical reasons.

The sample was introduced in a glass cylindrical cell $(15 \mathrm{~mm}$ diameter and $110 \mathrm{~mm}$ height) and analysed using a light beam emitted in near infrared ( $850 \mathrm{~nm}$ wavelength) which scanned the entire sedimentation column with a step of $40 \mu \mathrm{m}$. The time elapsed between two consecutive scans was chosen as a function of destabilization kinetics of suspension. Transmitted and backscattered lights were analysed using detectors placed, respectively, at $0^{\circ}$ and $135^{\circ}$ from the incident beam direction, so two evolutions of profiles versus times have been obtained: respectively, one in transmission ( $T$ ) and one in backscattering (BS). An example is show in Fig. 1, where we can differentiate three parts to consider in the sedimentation column (which correspond to the sample volume including between the special stopper and the meniscus):

- At the top of the sample, the variations of transmission profiles present a shift on the left of the clarification front, which indicated an increase of the supernatant volume. We can note that a similar variation exists in the backscattering profiles, but they result to secondary reflections of light on the glass cell. Therefore, in this case, one should only consider the transmission signals, and not the BS one.

- The middle of the column is too concentrated to have a transmission signal. So from this part we can study the stability or instability on the BS profiles evolution.

- At the bottom one, we can observe in Fig. 1, an increase of BS profiles values due to sediment formation (increase of $\phi_{v}$ value).

\subsection{Phase separation index calculation}

The phase separation index (PSI) was proposed in a previous sedimentation study [5]. It was calculated from backscattering profiles in the part of sediment formation. It was defined (Eq. (1)) by the product of relative sediment height at $15 \mathrm{~min}\left(H_{s} / H_{C}\right)$ and the average value of the percentage of backscattered light $\left(B_{S} \%\right)$ in the zone defined as the sediment (i.e. in $H_{s}$ in Fig. 1 ). The choice to measure these values at $15 \mathrm{~min}$ is arbitrary, but it seems to be a reasonable time to lead a settling analysis.

$\mathrm{PSI}=\left(\frac{H_{s}}{H_{c}}\right) \times\left(B_{s}^{\%}\right)$

with $H_{c}$, the sedimentation column height.

So, this index takes into account the quantity and dispersion state of matter which is present in sediment because $B_{S} \%$, the average backscattering percentage of the sediment, is directly linked to the particle volume fraction $\left(\phi_{v}\right)$ and the mean particle size $(d)$ as shown in Eqs. (2) and (3). As the sediment structure is directly link to mesostructural agglomeration state of particles during settling processes, the PSI can be a pertinent parameter to characterize suspension stability.

$$
\begin{aligned}
& B_{S}^{\%} \approx \frac{1}{\sqrt{\lambda *}} \\
& \lambda *\left(d, \Phi_{v}\right)=\frac{2 d}{3 \Phi_{v}(1-g) Q_{s}}
\end{aligned}
$$




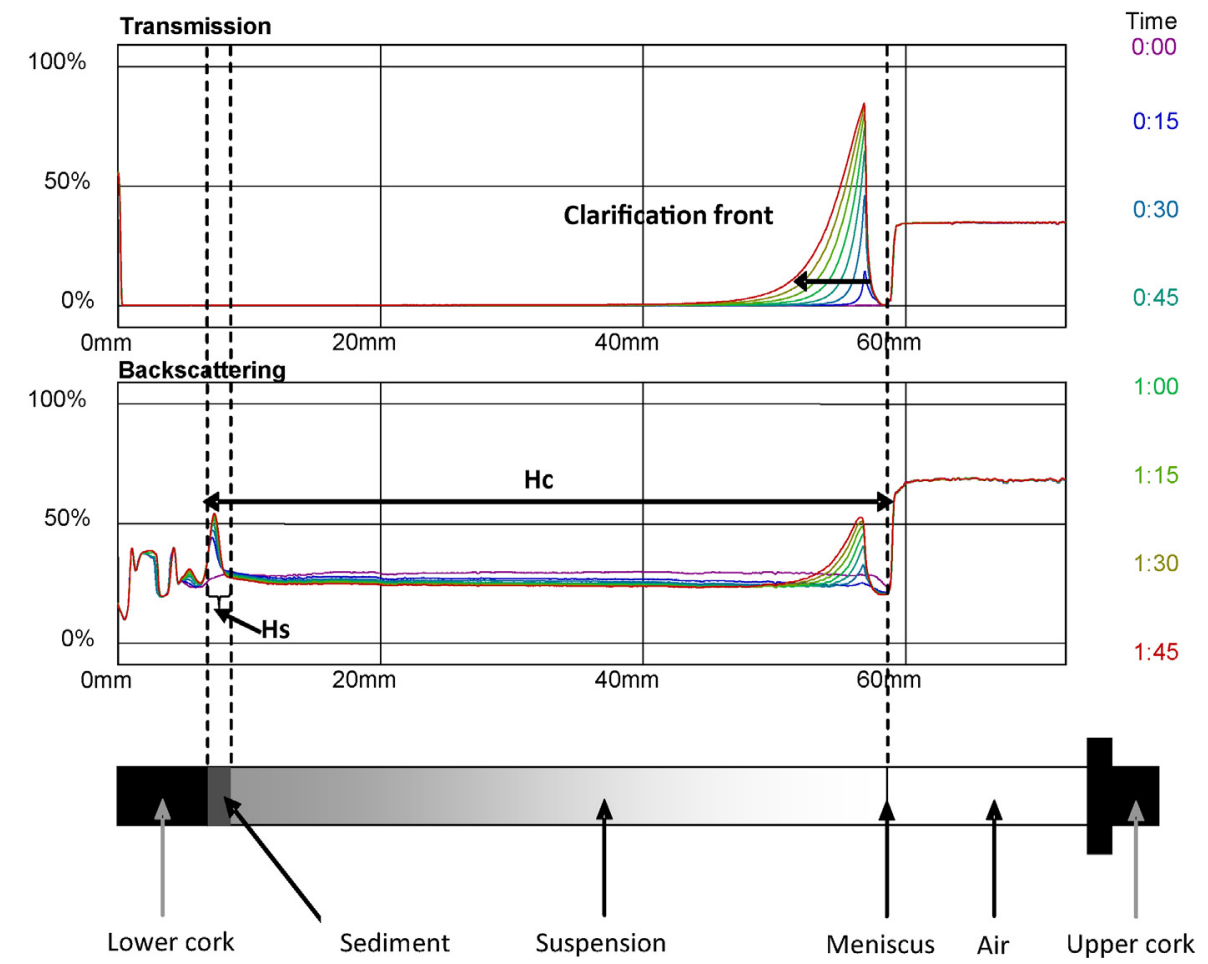

Fig. 1. Example of Turbiscan MA 2000 transmission and backscattering profiles. Determination of sedimentation column height $\left(H_{c}\right)$ and sediment height $\left(H_{s}\right)$.

With $\lambda^{*}$ : the photon transport length in the considered medium $(\mathrm{m}), Q_{s}$ : the scattering efficiency factor (dimensionless) and $g$ : asymmetry factor (dimensionless) in Lorenz-Mie theory.

\section{Results}

\subsection{Suspensions characteristics and influent parameters}

Suspension behaviours depend on numerous parameters which may be interdependent. Moreover, the phase separation index cannot evolve linearly as a function of these parameters as it is shown in Fig. 2.

To estimate the type of a suspension, solid mass fraction and nine others characteristics currently measured were chosen and presented in Table 1. Mass fraction represents the average distance between the particles in suspension. Density of particles, granular mode, granular spreading, shape index and specific surface are linked to the intrinsic properties of the solid particles. Electrophoretic mobility, $\mathrm{pH}$, ionic conductivity and surfactant concentration were chosen because of their great influence on the solid/liquid interphase properties.

In summary Table 1, it has been studied:

- Two different densities of solid,

- three pH (neutral, acid or basic) and three surfactant concentrations for kaolin and alumina suspensions (electrostatic and/or steric repulsion effects),

- four states of electrostatic dispersion controlled by electrophoretic mobility measurements,

and about granular characteristics:

- three granular populations (micronic particles or fine supracolloids, large supra-colloids, and settled particles),

- two shape factors,
- and four ranges of specific surface (in th order to $\mathrm{m}^{2} / \mathrm{g}, 0.1 \mathrm{~m}^{2} / \mathrm{g}$, $0.01 \mathrm{~m}^{2} / \mathrm{g}$ and $0.001 \mathrm{~m}^{2} / \mathrm{g}$ ).

\subsection{Typology of suspensions}

As the solid concentration of a suspension increases, three cases can be usually distinguished based on rheological properties evolution [11]. At low concentration, particles can be considered as isolated. At higher concentration, particles are closer and begin to develop significant hydrodynamic and physico-chemical interactions [12]. When concentration is further increased, particles or agglomerates are in contact. Predominant interaction will have a critical influence on the settling behaviour of these media too.

Taking into consideration the chosen powders and the formulation made, a separation into four classes appeared to be more appropriate [6]. These classes have been determined in function of PSI versus solid mass fraction evolutions (Fig. 2):

- Class I: diluted suspensions, where the sediment is constituted of non-agglomerated particles and occupies a small volume. Sedimentation rate is small and is almost independent of the concentration; and a concentration gradient takes place while settling process. This behaviour is diluted suspensions behaviour [3] where particles do not interact significantly through colloidal forces.

- Class II: concentrated suspensions of non-cohesive particles, which is constituted by suspensions in which particles are sufficiently close to generate steric interactions but do not develop significant attractive interactions.

- Class III: concentrated suspensions of cohesive particles, which is characterized by the existence of agglomerates due to attractive interactions. Forming large structures, the agglomerated particles generally exhibit high sedimentation rate [13]. The sedimentation mechanism changes radically and "hindered settling" regime occurs. This regime is characterized by a sharp boundary between the suspension and the supernatant [14]. The solids 


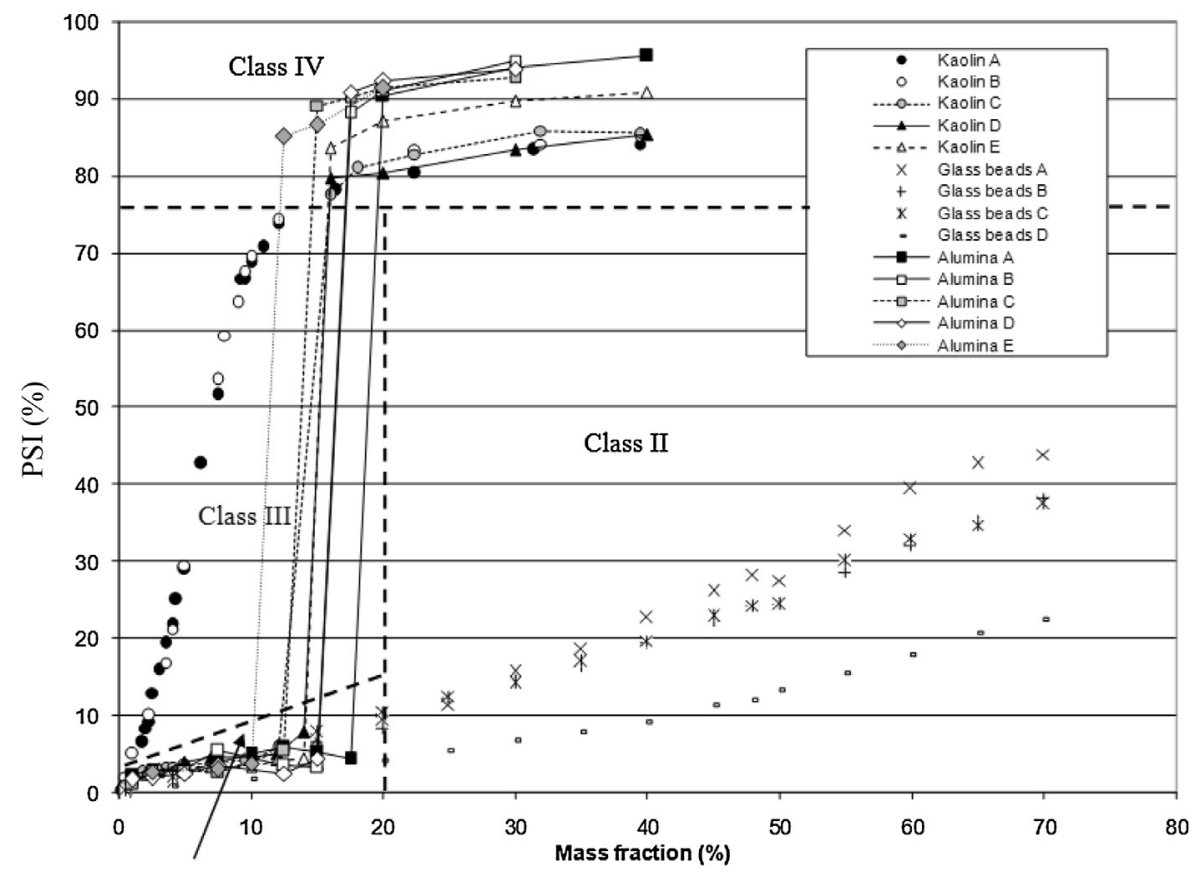

Class I

Fig. 2. Phase separation index variations as a function of mass fraction and different classes of suspension.

subside as a more or less consolidated structure due to the fact that concentration is large enough to develop agglomerate contact as they settle [15].

- Class IV: solid suspensions, in which all the agglomerates or unit particles form a contact network in the suspension. In this case settling phenomenon can occur only by compression. Sedimentation rates tend to zero because the weight of the particles in the samples is too small to create a compression force high enough to generate a significant movement in the suspension. Evolution in the PSI as function of mass fraction in this class is due to the increase of backscattering percentage value generated by the higher initial concentration.

Thereby, each class was associated to a prevailing type of particular interactions which are reported in Table 2.

\section{Neural networks for stability classification}

\subsection{Definition of neural networks}

Neural networks are generally used to identify complex behaviours not well understood or that cannot be described by algebra or rules (as in logic or fuzzy logic). Briefly these systems are based on parametric functions, called neurons, which calculate a non-linear transformation of their input variables. A neural network is defined, first, by the type of neurons and secondly by the network's architecture. The architecture is the scheme of connexion of neurons. An architecture called as multilayer perceptron is well known and provides the property of Universal Approximation which means that it is able to identify any non-linear and differentiable function [16]. This architecture is composed of one output layer and one hidden layer. Each layer contains neurons, and neurons belonging to the same layer have no interactions one with the others. Moreover, it had been shown that multilayer perceptrons are parsimonious because they generally need fewer coefficients than other statistical models [17]. Once the network is created, appropriated values of the parameters must be computed. This step is the training phase. Once this phase is achieved, the model quality has to be evaluated in a test phase. More detailed presentation of neural networks can be found in [8].

\subsection{Training phase}

During the training phase, examples with known inputs and outputs variables are presented to the network. They constitute the training set. These examples must be numerous and as diverse as possible in order to represent the whole of possible behaviours. During training a cost function, here the squared error (Eq. (4)) between the estimated output and the measured one, for each example of the training set, is minimized.

$J=\frac{1}{2} \sum_{(k)}\left(y(\mathbf{C}, k)-y^{m}(k)\right)^{2}$

where $J$ is the cost function, $k$ corresponds to an input-output couple of variables, $y(\mathbf{C}, k)$ is the output variable $k, y^{m}$ is the measured output value $k$, and $\mathbf{C}$ is the matrix of parameters.

The training rule used in this study is the Levenberg-Marquardt rule. It has been chosen because of its high efficiency [18,19]. This learning rule generally leads in a few iterations to a very satisfactory solution.

\subsection{Complexity adjustment}

It has been shown [20] that the error calculated such as expressed in Eq. (4) over the training set, is not a good estimator of the generalization error. Because of overfitting which is caused by over-parameterization (too many parameters in the model) the training specializes the model over the training set and is unable to generalize to unknown set of data: the test set [21]. This is known as the biais-variance dilemma.

In order to prevent over-parameterization, it is thus important that the network would be as parsimonious as possible, and, taking into account the available set of data, to choose the more parsimonious model as possible [22]. 


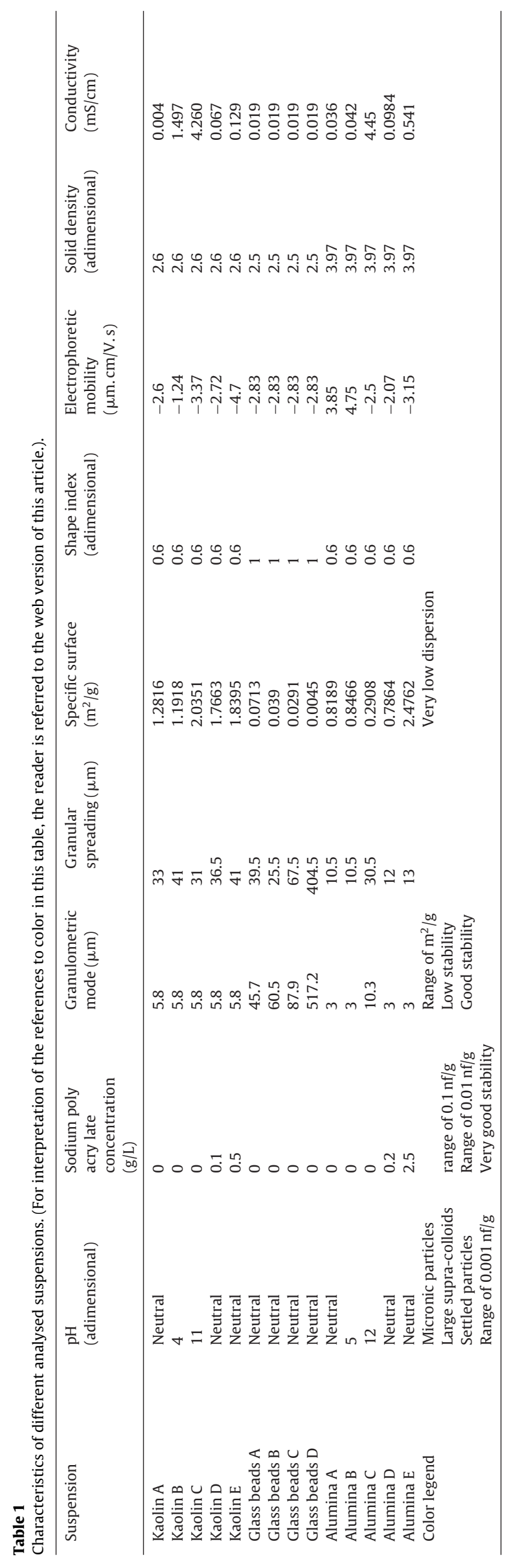

Table 2

Classes and prevalent interparticle interactions.

\begin{tabular}{ll}
\hline Class & Interparticle prevalent interactions \\
\hline Class I & No significant interaction \\
Class II & Steric interactions \\
Class III & Physico-chemical interactions \\
Class IV & Contact network \\
\hline
\end{tabular}
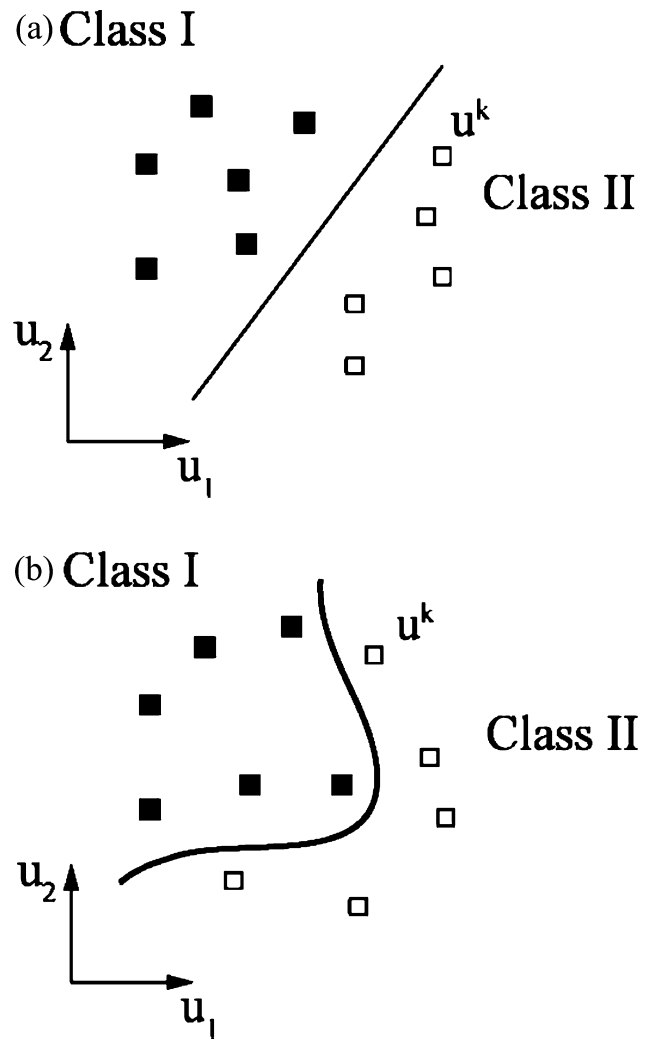

Fig. 3. (a) Classes are well represented; examples $\left\{u^{k}\right\}$ are linearly separable (b) examples $\left\{u^{k}\right\}$ are not linearly separable. Representation of classes is good but less effective than in previous configuration.

For this reason, several methods of variable selection and complexity selection have been proposed [8].

\subsection{Generalization/test}

The aim of this step is to evaluate the ability of the network to generalize what it has learned to new examples never encountered before. For that, the test step consists in presenting examples that have not been used during the training phase. These new examples compose the test set. Each test example is presented to the network and the values of the output variables are compared with the expected one according to the Eq. (4).

\subsection{Neural networks based classification}

The classification task is the assignment of a code to an input variable vector. Two configurations can be encountered:

- Examples can be separated by a linear function as illustrated in Fig. 3a. The set of data is called as linearly separable and the task can be addressed by linear methods, in particular with a single neuron (Fig. 4). A single neuron computes first its potential, which is the weighted sum of its inputs for the example $k$ : 


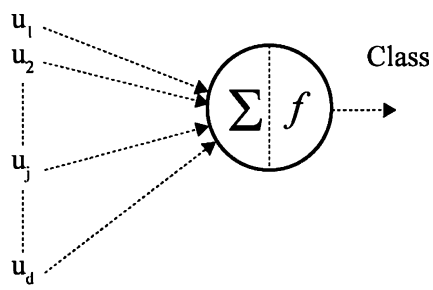

Fig. 4. A single neuron as linear classifier, $f($.$) is the threshold function.$

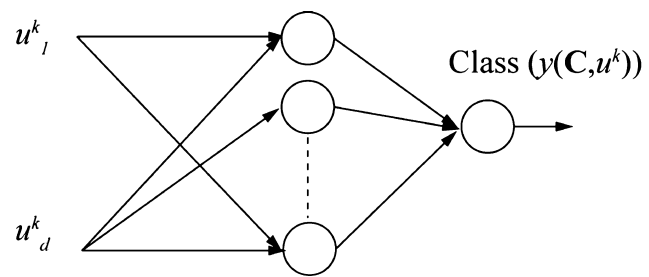

Fig. 5. Multilayer perceptron with one hidden layer and one output neuron.

$x^{k}=\sum_{j} c_{j} u_{j}^{k}$. Second, the single neuron evaluates its class by a threshold function $(f()=$. heaviside function $)$.

- Examples cannot be separated by a linear function as shown in Fig. 3b. The set of data is called as non-linearly separable and requires a multilayered neural network.

The multilayer perceptron is composed of two layers of neurons as shown in Fig. 5. Each neuron is a parametric function, which computes:

$y(\mathbf{C}, k)=f\left(x^{k}\left(\mathbf{C}, u^{k}\right)\right)$

where $f($.$) is a sigmoid function whose preferred expression is$ $f\left(\mathbf{C}, u^{k}\right)=1 / 1-e^{x^{k}}$, with $x^{k}=\sum_{j} c_{j} u_{j}^{k}$ also called potential.

This kind of network is very famous and called "multilayer perceptron"; it is able of universal approximation (Section 3.3).

The ability to separate linearly two classes depends strongly on the representation of the classes, and by this way, on the choice of the variables, or features.

\section{Sedimentation behaviour classification}

The efficiency of neural networks modelling has been proved in various domains as robotics, signal or image processing, and also in natural sciences, as water sciences [23], optimization of the dimensioning of filtration processes [24], or in Physical sciences. Useful applications of these systems have been also carried out in suspensions domain [25].

\subsection{Data coding}

As described in the introduction, experimental measurements led to the determination of four types of sedimentation. These types defined four classes in which each example of suspension must be classified. For that, a classical and efficient coding was used. It is a disjunctive coding, using as much digit as classes to code: when an example belongs to a particular class its output value is coded as 1 for the digit associated to this class, and - 1 for the other digits. Thus, as illustrated in Table 4 "example a" belongs to class I, "example b" belongs to class II, and so on. Each class is thus represented by one particular output of the classifier.

This choice was preferred because it is known that in the case of a classification problem with $n_{c}$ classes, it is better to have $n_{c}$ binary outputs than only one with $n_{c}$ levels [26]. Moreover this
Table 3

Coding classes.

\begin{tabular}{lllll}
\hline & Digit 1 & Digit 2 & Digit 3 & Digit 4 \\
\hline $\begin{array}{l}\text { Example belonging to } \\
\text { class I }\end{array}$ & +1 & -1 & -1 & -1 \\
$\begin{array}{l}\text { Example belonging to } \\
\quad \text { class II }\end{array}$ & -1 & +1 & -1 & -1 \\
$\begin{array}{c}\text { Example belonging to } \\
\text { class III }\end{array}$ & -1 & -1 & -1 & -1 \\
$\begin{array}{l}\text { Example belonging to } \\
\text { class IV }\end{array}$ & -1 & -1 & -1 & +1 \\
$\begin{array}{c}\text { Confusion between (for } \\
\quad \text { example) classes I } \\
\text { and III }\end{array}$ & 1 & -1 & 1 & -1 \\
$\begin{array}{l}\text { Unknown example (no } \\
\text { class is recognized) }\end{array}$ & -1 & -1 & -1 & -1 \\
\hline
\end{tabular}

Table 4

Linear separability of classes.

\begin{tabular}{ll}
\hline Class I (no significant interaction) & No separable from the others \\
\hline Class II (steric interactions) & Separable from the others \\
Class III (physico-chemical interactions) & No separable from the others \\
Class IV (contact network) & Separable from the others \\
\hline
\end{tabular}

coding enables the classifier having more possible responses than the number of classes: $2^{n_{c}}$ states are available with this coding. The classifier is thus able to indicate difficulties in classification, for example if two output variables respond that the input belongs to their (different) classes, one can interpret this configuration as a confusion between the two specific classes. By this way, the misclassification rate is diminished, and the non-classified rate is increased.

\subsection{Database}

The database consisted of 189 examples. Each one corresponded to an experiment of suspension behaviour. Inputs variables consisted of a vector of 10 characteristics of the suspension (nine listed in Table 3 and solid mass fraction). Preprocessing must be performed on these variables [8]. In this application the most relevant transformation is the normalization between 0 and 1 for all the variables except for the mass fraction, which is subjected to a non-linear transformation before to be applied to the network. This transformation was the log function, it thus allowed the separation between classes (as shown hereafter) to be linear.

\subsection{Evaluation of task difficulty: are the examples linearly separable?}

According to the presentation of Section 4, it is necessary to identify the difficulty of the classification, in order to adopt the more parsimonious network at each step of the study. It is thus necessary to assess if the classes, represented by the database, are linearly separable.

For that, the training must operate on the complete database using only one linear neuron [27]: using this method, when the training converges, examples are separable; if not, they are not separable.

Table 4

Four networks where thus trained each one to estimated one digit of the code of the classes. Each network was based on a single neuron (Section 4.5, Fig. 4), and trained with the perceptron rule [26]. The result, shown in Table 5 is that only two classes over four are linearly separable from the others. For the classes II and IV which are linearly separable from all the others, the next step is thus to assess the generalization capability of the classifier; for 
Table 5

Values of parameters linking each variable to the network, for each class.

\begin{tabular}{llllc}
\hline & Class I & Class II & Class III & Class IV \\
\hline Solid mass fraction & 18 & 11 & 9.2 & 19.5 \\
Solid density & 7.8 & 6.1 & 6.6 & 1 \\
Granulometric mode & 4.6 & 0.4 & 3.6 & 2.4 \\
Granular spreading (d95-d5) & 4.1 & 0.2 & 1.4 & 0.4 \\
Shape factor & 6.7 & 1.9 & 1.8 & 19.1 \\
Specific surface & 7.8 & 7.1 & 2.6 & 1.2 \\
pH & 19 & 3.2 & 14.6 & 4.2 \\
Surfactant concentration & 8.9 & 0.5 & 13.3 & 1.8 \\
Electrophoretic mobility & 6 & 0.5 & 5 & 3.2 \\
Conductivity & 5.92 & 3.19 & 1.39 & 1.63 \\
\hline
\end{tabular}

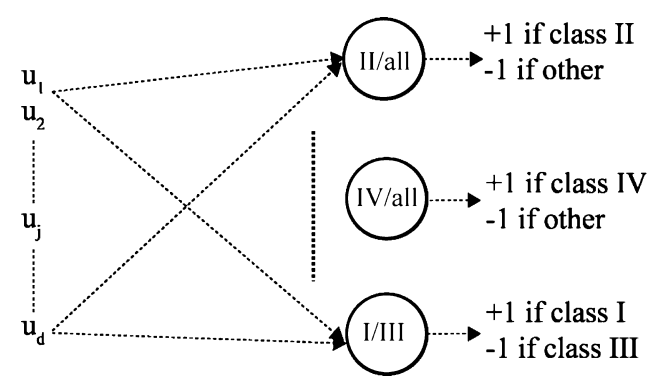

Fig. 6. Architecture of the networks inferred by the STEPNET procedure.

the classes I and III, which are not linearly separable, one from all the others, it is necessary to increase the complexity of the separation. For that two methods are investigated: first a stepwise procedure, which assesses the separability of classes by pairs, secondly, if linear separation by pair is impossible, the implementation of a multilayer perceptron, which assumes the property of universal approximation.

\subsection{Pairwise separation of the classes I and III}

Still using the architecture with one layer of parameters showed in Fig. 4, we proposed to follow the "divide and conquer" strategy explained in [28]: the STEPNET procedure. In this procedure, after the assessment of the separability of the classes one from the others, the classes which were not separable (here the classes I and III) were attempted to be separated one from each other by a pairwise linear separation. Thus, for each studied separation, only examples of the two considered classes were used and tested.

Considering that separations between classes II and all the others, and between class IV and all the others were already done by the method described in Section 5.3, only the pairwise separation between the classes I and III needed to be studied.

In this case, only examples which corresponded to class I and III were retained (74 examples) and a "one layer perceptron" was used. The result was that these examples were linearly separable.

At the end, the linear separability of the four classes was assessed (pairwise or not pairwise), using the STEPNET method, with the architecture presented in Fig. 6.

\subsection{Generalization ability}

As the linear separability (pairwise or not pairwise) of all the classes was proved, and in order to maximize the performance of the classifier in generalization, we preferred evaluate the generalization only with pairwise separations. One can thus easily understand, as illustrated in Fig. 7 that three straights lines implementing the three separations between one class and the three others are less constraining that only one linear separation and can thus better separate two classes.
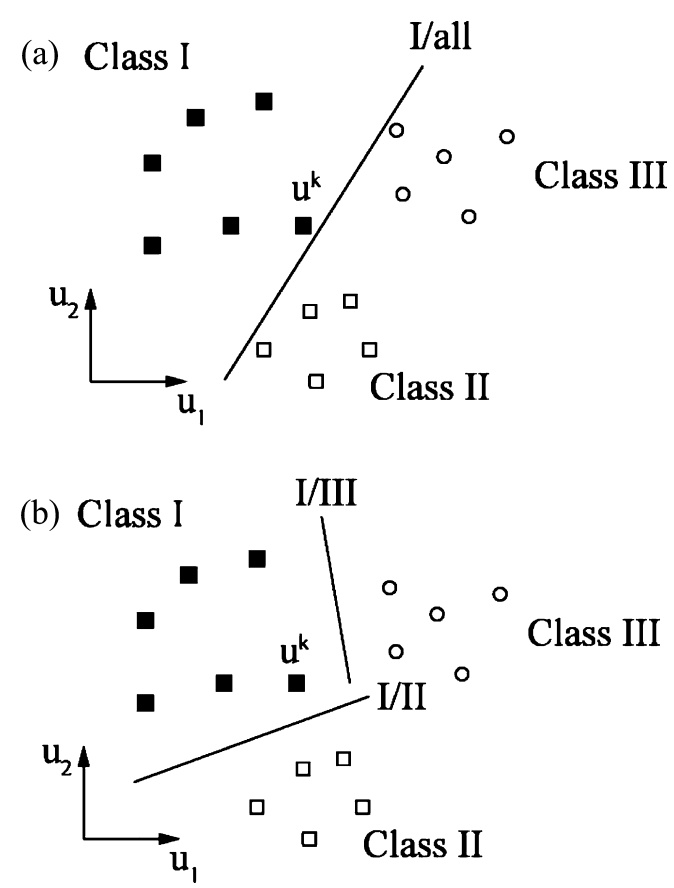

Fig. 7. (a) Linear separation from one class (I) to all others (II and III). Separation is very close to the examples and generalization could be not perfect. (b) Linear separation from one class (I) to each of others (II and III). Separations can be optimal.

The pairwise procedure was applied to six elementary "one layer perceptron" in order to implement the separations: each class from each other as illustrated on Fig. 8.

The generalization ability was assessed on a set which was not used in training. Various test sets where used consisting of $20 \%, 40 \%$ and $60 \%$ of the database. For each of these configurations the training set, respectively, consisted of $80 \%, 60 \%$ and $40 \%$ of the database. After the training of each perceptron by the Levenberg-Marquardt training rule, computing each linear separation (Fig. 8), it appeared first that all the separations where achieved, and secondly that $100 \%$ of good classification was obtained on both training and test sets, for all the partitions of the database.

The classification, operated by the pairwise procedure was thus very efficient an improve significantly the good classification rate.

Moreover, it is interesting to note that the STEPNET procedure do not provide a linear model because the classification was proceeded in two steps: first the classification by pair and after that a logical function in order to extract the class. It is thus considered that a non-linear classification is made as with it could be the multilayer perceptron. This classification is done using several very parsimonious networks, assuming thus a maximization of the generalization capability. Of course this promising result does not presume that such good classification would be reached on real and noisy data. It encourages proceeding to the pairwise STEPNET procedure rather than in standard multilayer perceptron to perform classification of suspensions.

\section{Validation of the model: Analyse of parameters values}

In order to verify the relevance of the model proposed in this work to classify suspensions, the parameters linking each input variable to the hidden layer was compared and linked to suspension characteristics, as it could influence suspension behaviour and mesostructural organization observed.

As it have been shown in a previous study [6] carried out in order to obtain a similar classification approach realized with 


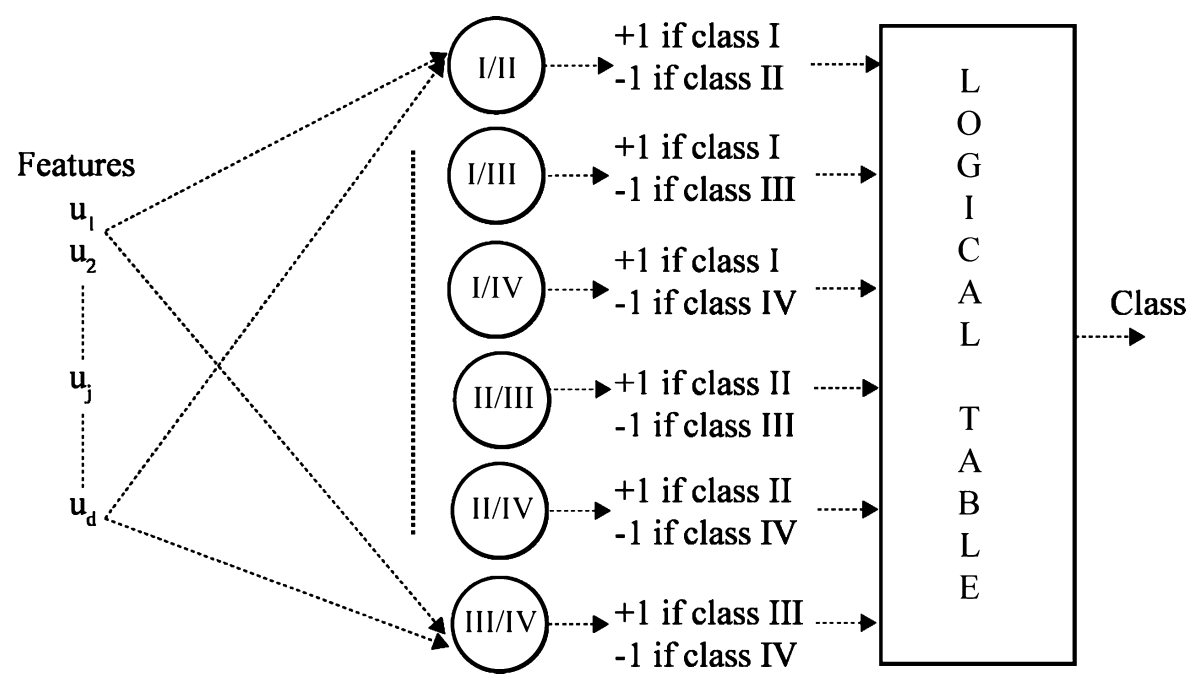

Fig. 8. Non linear classification using six linear perceptrons and a logical table.

backpropagation algorithm, the model parameters linked to each input variable are reported in Table 5.

In accordance with all bibliographical data and experiments related to these kinds of media, mass fraction is a key parameter in stability, regardless of the class of suspension taken into account $[2-5,12,13]$. Actually, one may reasonably consider that higher absolute value of parameters reflects a major influence of the corresponding parameter. When the other 9 inputs are studied, their relative influence can show (see the shaded cells in Table 5):

- the importance of particle solid density for the class I, II and III for which the settling phenomena can occur (not in class IV where the particles forming a network of contacts),

- the importance of $\mathrm{pH}$, surfactant concentration and electrophoretic mobility for the class I and III for which the agglomeration/dispersion phenomena can occur (not in class II where the particles have no physicochemical interaction),

- the increasing importance of the specific surface to class IV to I,

- and an higher importance of particle granular characteristics (granulometric mode, granular spreading and shape factor) for the class I, except for the class IV where the shape factor is particularly high, that could not be explained by this work.

As a conclusion of this study, once can state that parameters values are consistent with physicochemical behaviour or mesostructure organization of each class of suspension. This indicates that the classification established through neural networks is coherent with the knowledge of these systems.

\section{Conclusion}

The aim of this work was to extend the classification made with kaolin particle using phase separation index that has been proposed in a previous work [5], to other mineral suspensions in order to determine their prevailing type of interparticle interactions and to estimate their stability regarding the sedimentation phenomenon. In order to achieve this, a classification through neural networks has been carried out. Ten discriminatory variables were used: mass fraction, density of particles, granular mode, granular spreading, shape index, electrophoretic mobility, specific surface, $\mathrm{pH}$, surfactant concentration and ionic conductivity. To maximize the capability of generalization of the neural-based model, the more parsimonious architecture was preferred. Thus, after having assessed the complexity of the classification, an architecture composed of six linear perceptrons was deduced by the STEPNET procedure. Generalization was assessed on various sets of test sets up to $60 \%$ of the database. In all these configurations $100 \%$ of good classification was obtained over the considered test sets. These very satisfying results validate the proposed method: prevailing type of interparticle interactions and thus their stability can be known from a priori variables.

\section{References}

[1] P. Coussot, Rheometry of Pastes Suspensions and Granular Media: Applications in Industry and Environment, Wiley, Hoboken, NJ, 2005

[2] Th.F. Tadros, Correlation of viscoelastic properties of stable and flocculated suspensions with their interparticle interactions, Adv. Colloid Interface Sci. 68 (1996) 97-200

[3] P. Grassia, S.P. Usher, P.J. Scales, A simplified parameter extraction technique using batch settling data to estimate suspension material properties in dewatering applications, Chem. Eng. Sci. 63 (2008) 1971-1986.

[4] S. Diehl, Estimation of the batch-settling flux function for an ideal suspension from only two experiments, Chem. Eng. Sci. 62 (2007) 4589-4601.

[5] R. Vie, N. Azéma, J.C. Quantin, E. Touraud, M. Fouletier, Study of suspension settling: a new approach to determine suspension typology and particle interaction, Colloids Surf., A: Physicochem. Eng. Aspects 298 (2007) 192-200.

[6] R. Vié, Approche globale de caractérisation des suspensions: stabilité, classification, modélisation, in: Ph.D. in Materials Chemistry, Ecoles des Mines d'Alès /Université Montpellier II, 2006.

[7] O. Mengual, G. Meunier, I. Cayré, K.P. Puech, Snabre TURBISCAN MA 2000 multiple light scattering measurements for concentrated emulsion and suspension stability analysis, Talanta 50 (1999) 445-456.

[8] G. Dreyfus, Neural Networks Methodology and Applications, Springer, Berlin, 2004.

[9] G. Artigue, A. Johannet, V. Borrell, S. Pistre, Flash flood forecasting in poorly gauged basins using neural networks: case study of the Gardon de Mialet basin (southern France), Nat. Hazard. Earth Syst. Sci. 12 (2012) 3307-3324.

[10] Fine a-alumina particle, United States Patent Application 20070021292.

[11] P. Coussot, C. Ancey, Rheophysique des pâtes et des suspensions, EDP Sciences, Les Ulis, 1999.

[12] F. Tharwat, Tadros, Dispersion of Powders in Liquids and Stabilization of Suspensions, Wiley-VCH, 2012

[13] B. Fitch, Sedimentation of flocculent suspensions: state of the art, AIChE J. 25 (1979) 913-930.

[14] B. Fitch, Sedimentation process fundamentals, Trans. Am. Inst. Electr. Eng. 223 (1962) 129-137.

[15] J.R. Karl, S.A. Wells, Numerical model of sedimentation/thickening with inertial effects, J. Environ. Eng.-ASCE 125 (1999) 792-806.

[16] K. Hornic, M. Stinchcombe, H. White, Multilayer feedforward network are universal approximators, Neural Networks 2 (1989) 359-366.

[17] A.R. Barron, Approximation bounds for superpositions of a Sigmoidal function, IEEE Trans. Inf. Theory 39 (1993) 3.

[18] M.T. Hagan, M.B. Menhaj, Training feedforward networks with the Marquardt algorithm, IEEE Trans. Neural Networks 5 (1994) 989-993.

[19] W.H. Press, S.A. Teukolsky, W.T. Vetterling, B.P. Flannery, Numerical Recipes in C. Cambridge University Press, 1992.

[20] S. Geman, E. Bienenstock, R. Doursat, Neural networks and the bias/variance dilemma, Neural Comput. 4 (1992) 1-58. 
[21] L. Kong-A-Siou, A. Johannet, V. Borrell, S. Pistre, Optimization of the generalization capability for rainfall-runoff modeling by neural networks: the case of the Lez aquifer (southern France), Environ. Earth Sci. 65 (8) (2012) 2365-2375

[22] L. Kong-A-Siou, A. Johannet, V. Borrell, S. Pistre, Complexity selection of a neural network model for karst flood forecasting: the case of the Lez Basin (southern France), J. Hydrol. 403 (2011) 367-380.

[23] M. Toukourou, A. Johannet, G. Dreyfus, P.A. Ayral, Rainfall-runoff modeling of flash floods in the absence of rainfall forecasts: the case of "Cévenol flash floods", Appl. Intell. 35 (2) (2011) 178-189.

[24] A. Cougnaud, C. Faur, P. Le Cloirec, Modélisation de l'adsorption dynamique de pesticides sur filtre à charbon actif à l'aide de réseaux de neurones, in: Article Presented at the Conference: Récents Progrès en Génie des procédés, 2005.
[25] T.Y. Pai, Y.P. Tsai, H.M. Lo, C.H. Tsai, C.Y. Lin, Grey and neural network prediction of suspended solids and chemical oxygen demand in hospital wastewater treatment plant effluent, Comput. Chem. Eng. 31 (2007) 1272-1281.

[26] S. Knerr, L. Personnaz, G. Dreyfus, Single layer learning revisited: a stepwise procedure for building and training a neural network, in: J. Fogelman (Eds.), Neurocomputing: Algorithms, Architectures and Applications, Springer-Verlag Inc., Berlin, pp. 41-50.

[27] R.O. Duda, P.E. Hart, Pattern Classification and Scene Analysis, Wiley, New York, NY, 1973.

[28] S. Knerr, L. Personnaz, G. Dreyfus, Handwritten digit recognition by neura networks with Single-layer training, IEEE Trans. Neural Networks 3 (1992) 962-968. 